\title{
SOME NEW VIEWPOINTS IN DIFFERENTIAL GEOMETRY IN THE LARGE ${ }^{1}$
}

\author{
SHIING-SHEN CHERN
}

1. Introduction. Differential geometry in the large is concerned with relations which exist between the local properties of a geometric being $^{2}$ given in a manifold and the properties of the manifold as a whole. The manifold is differentiable in the sense that it is covered by a set of coordinate neighborhoods, each having the same number of coordinates, and that two different systems of coordinates in a common region are related by a differentiable transformation of class not less than 1. The latter assumption allows the use of differentiation in studying the local geometry and thus leads to a number of geometric properties the study of which was initiated by Euler, Gauss, and Monge.

To give an example of a problem of this nature we consider a closed surface $S$, differentiably imbedded (of class not less than 2 ) in a Euclidean space of three dimensions. Let $K$ be the Gaussian curvature and $d A$ the surface element of $S$. Then the classical GaussBonnet formula asserts that

$$
\frac{1}{2 \pi} \iint_{S} K d A=2(1-p),
$$

where $p$ is the genus of $S$. This formula expresses the genus $p$ of $S$, a topological invariant, in terms of a differential invariant. In other words, $p$ is completely determined by the local properties of $S$.

As another example consider a closed curve $C$ imbedded in the Euclidean plane. If $C$ is rectifiable with length $l$ and bounds a region of area $A$, then

$$
l^{2}-4 \pi A \geqq 0 .
$$

The equality sign holds only when $C$ is a circle. This so-called isoperimetric inequality has recently been derived in an unexpected

An address delivered before the Summer Meeting of the Society in New Brunswick on September 15, 1945, by invitation of the Program Committee; received by the editors June 22, 1945.

1 Dedicated to Professor Elie Cartan.

2 The term geometric object was first suggested by Professor Oswald Veblen, who now prefers to use geometric being, a translation of the French term "être géometrique" due to Cartan. 
way in integral geometry, which deals with the integrals of differential invariants.

In problems of this nature there are two aspects, a local one and a global one. During several decades of intensive work the local aspect has been extensively studied and developed, culminating in the doctrine of tensor analysis. While tensor analysis gives an adequate tool for handling most local problems, it is natural that the study of global problems will necessitate the introduction of new concepts and the modifications of the classical treatment which has so far been followed. It is the main aim of the present article to emphasize the importance, for the global study of manifolds with a geometric being, of drawing into consideration new topological spaces associated with the manifold. In fact, this idea is important for both local and global aspects of differential geometry. For local problems it is certainly very familiar to Elie Cartan, who introduced the notion of tangent space ("espace tangent") for his general theory of geometric beings (affine connections, projective connections, conformal connections, and so on). The tangent space in the sense of Cartan is not always the space of tangent vectors and therefore constitutes one of the sources of difficulty for the understanding of his work. On the other hand, recent works on fibre bundles in topology (Stiefel, Whitney, Feldbau, Ehresmann, Pontrjagin, Steenrod, and so on) seem to lay the foundation for a global theory of the ideas of Cartan. It is a conviction of the author that a mingling of these two streams of thought will give the ideas and tools better adaptable to the study of differential geometry in the large than hitherto achieved. The present article will be devoted to a discussion of different aspects of the problem arising from this viewpoint.

Before going into details, we shall give a brief summary of the main points of our discussion. Our problem will be the study, on a differentiable manifold, of a geometric being given in terms of each local coordinate system by components which obey a definite transformation law under change of the local coordinates. We emphasize that for each such problem it is in general possible to define in a certain sense a natural fibre bundle associated with the manifold. The geometric being then defines in a unique way a set of linear differential forms in the fibre bundle, which gives all the local properties of the geometric being. For Riemannian manifolds the natural fibre bundle is the space of all frames on the manifold and the corresponding linear differential forms give what is essentially known as the parallelism of Levi-Civita. The nature of the fibre bundle to be associated is best decided by the solution of the so-called problem of 
equivalence, which is the form problem in the case of Riemannian geometry. With the set of linear differential forms in the fibre bundle, differential forms of higher degree can be obtained by operations of Grassmann analysis. Such differential forms define cochains and also cocycles, when they are exact. A study of the interrelations between the cochains and cocycles in the given manifold and the associated fibre bundles, which arise from the projection of the latter into the former, will give a deeper understanding of the global theory of the geometric being. An attempt is made in the following to illustrate in the simplest cases the results that can be derived from this idea. Although this will be our main concern in the present paper, it does not imply that this is the only service that the theory of fibre bundles could render to differential geometry. In fact, there are indications that other possible applications could be fruitfully made. The whole field seems therefore to deserve a more thorough exploitation.

2. Grassmann algebra. At the fore of the Cartan scheme is the so-called Grassmann algebra. In a formal algebraic way this can be defined as follows: Let $K$ be a field of characteristic zero and let $V(n, K)$ be a vector space of $n$ dimensions over $K$. The Grassmann algebra $H$ over $V(n, K)$ is a hypercomplex system over $K$ satisfying the following conditions:

(1) $H$ contains a unit element 1 and all elements of $V(n, K)$ and is generated by these elements (by operations of the hypercomplex system).

(2) If $x, y$ belong to $V(n, K)$, their multiplication satisfies the alternating rule:

$$
x y=-y x .
$$

(3) The elements of $H$ satisfy no other relations than those derived from (1) and (2).

Let $e_{1}, \cdots, e_{n}$ be a set of basis elements of $V(n, K)$. From the above conditions the following conclusion can be drawn: Every element of $H$ can be expressed in one and only one way as a linear combination, with coefficients in $K$, of the elements $1, e_{i_{1}} \cdots e_{i_{m}}$, $i_{1}<\cdots<i_{m}, i_{1}, \cdots, i_{m}=1, \cdots, n$. In symbols we write, if $x$ belongs to $H$,

where

$$
x=\sum_{m=0}^{n} x^{(m)},
$$

$$
x^{(m)}=\sum_{i_{1}, \cdots, i_{m}=1 ; i_{1}<\cdots<i_{m}}^{n} a_{i_{1} \cdots i_{m}} e_{i_{1}} \cdots e_{i_{m}} .
$$


The element $x$ is said to be of degree $m$, if

$$
x^{(m)} \neq 0, \quad x^{(m+1)}=\cdots=x^{(n)}=0 .
$$

It is called an exterior form, or simply a form, if $x^{(i)}=0, i \neq m$. The degree of an element is independent of the choice of the set of basis elements, and the same is true of the property that an element be a form.

Let $W$ be a vector subspace of dimension $m$ of $V(n, K)$. We take a set of basis vectors $f_{1}, \cdots, f_{m}$ in $W$ and form the product $w=f_{1} \cdots f_{m}$. It is easy to prove that $w$ is determined by $W$ up to a nonzero factor of $K$. The form $w$ is called the associated form of $W$. If $w_{1}$ and $w_{2}$ are the associated forms of two vector subspaces $W_{1}$ and $W_{2}$, of degrees $m$ and $p$ respectively, then $W_{1}$ and $W_{2}$ have a nonzero intersection when and only when $w_{1} w_{2}=0$. Moreover, if $w_{1} w_{2} \neq 0$, it is the associated form of the ambient vector subspace of dimension $m+p$ of $W_{1}, W_{2}$. Such are simple instances of ways in which the Grassmann algebra can be applied to the study of the geometry in a vector space.

3. The differential calculus of Elie Cartan. The Grassmann algebra was applied with success to the Pfaffian problem by Frobenius and Darboux. The so-called bilinear covariant is an exterior differential form of degree two. But it was Elie Cartan who started the systematic use of a calculus closely related to the Grassmann algebra and pertaining to exterior differential forms of higher degree.

Let $M^{n}$ be a differentiable manifold of dimension $n$ and class not less than 2. At a point $P$ of $M^{n}$ the contravariant vectors and the covariant vectors constitute two vector spaces (over the real field) which are dual to each other in the sense that one is the space of the linear forms in the other. The notion of a linear differential form at $P$ is identical with that of a covariant vector. For let $x^{1}, \cdots, x^{n}$ be local coordinates at $P$. With respect to the coordinates $x^{i}$ a covariant vector will have the components $X_{i}$. The differential form

$$
\omega=\sum_{i=1}^{n} X_{i} d x^{i}
$$

is then intrinsic in the sense that it is independent of the choice of the local coordinate system. Conversely, an intrinsic form $\omega$ has a set of components in each local coordinate system, which constitute a covariant vector.

Over the vector space of linear differential forms at a point $P$ of $M^{n}$ we construct the Grassmann algebra. A form of this Grassmann algebra is called an exterior differential form or simply a differential 
form. In a local coordinate system $x^{i}$ an exterior differential form can be written

$$
\omega=\sum_{i_{1}, \cdots, i_{m}=1}^{n} a_{i_{1} \cdots i_{m}}\left(x^{1}, \cdots, x^{n}\right) d x^{i_{1}} \cdots d x^{i_{m}},
$$

where we can assume the coefficients $a_{i_{1}} \ldots i_{m}$ to be skew-symmetric in their indices. In the terminology of tensor analysis the components $a_{i_{1}} \ldots i_{m}$ are the components of an alternating covariant tensor of order $m$.

In a neighborhood of $P$ in which the local coordinates $x^{i}$ are valid let $a_{i_{1}} \ldots i_{m}$ be of class not less than 2 . We define a differential form of degree $m+1$, called the exterior derivative of $\omega$, by means of the relation

$$
d \omega=\sum_{i_{1}, \cdots, i_{m}=1}^{n} d a_{i_{1}} \cdots i_{m} d x^{i_{1}} \cdots d x^{i_{m}}
$$

where $d a_{i_{1}} \ldots i_{m}$ is an ordinary differential. This process of exterior differentiation has the following properties, which can be easily verified:

(1) If $\omega, \theta$ are two differential forms of degrees $m$ and $p$ respectively, then

$$
d(\omega \theta)=d \omega \cdot \theta+(-1)^{m} \omega d \theta .
$$

(2) For any $\omega$ :

$$
d(d \omega)=0 .
$$

(3) The process of exterior differentiation is invariant under coordinate substitutions, by which we mean the following: Let the local coordinates $x^{i}$ in a neighborhood of $P$ represent a set of points $S$ in an open set $D$ in a Euclidean space of $n$ dimensions. Let an open set $E$ in a Euclidean space of $r$ dimensions with the coordinates $y^{1}, \cdots, y^{r}$ be mapped into $D$ by the mapping

$$
x^{i}=f^{i}\left(y^{1}, \cdots, y^{r}\right), \quad i=1, \cdots, n,
$$

such that $S$ is the image of a set $T \subset E$, the functions $f^{i}$ being supposed to be of class not less than 2. By the definition

$$
F\left(x^{1}, \cdots, x^{n}\right) \rightarrow F\left(x^{1}(y), \cdots, x^{n}(y)\right), \quad d x^{i} \rightarrow \sum_{k=1}^{r} \frac{\partial x^{i}}{\partial y^{k}} d y^{k} ;
$$

this mapping induces in a natural way (that is, with the multiplication of the Grassmann algebra preserved) a mapping of a differential 
form $\omega$ into a form of the space $\left(y^{1}, \cdots, y^{r}\right)$, which we denote by $\tilde{\omega}$. Then we have

$$
d \tilde{\omega}=\widetilde{d \omega} .
$$

In particular, when $r=n$, this signifies that the process of exterior differentiation is intrinsic, that is, independent of the local coordinate system under which it is performed.

The exterior differential forms are the forms under the integral sign in the theory of multiple integrals. In fact, let $\omega$ be a form of degree $m$ defined on the manifold $M^{n}$. Let $K^{m}$ be a chain of dimension $m$ (in the sense of combinatorial topology) on $M^{n}$, which is a sum, with integral, rational, or real coefficients, of images of simplexes in a Euclidean space by mappings which are differentiable of class not less than 2. Then it is possible to define the integral of $\omega$ over $K^{m}$. If $\partial K^{m+1}$ denotes the boundary of a chain $K^{m+1}$ of dimension $m+1$, the theorem of Stokes can be written

$$
\int_{\partial K^{m+1}} \omega=\int_{K^{m+1},} d \omega .
$$

To see the connection between this formulation of the Stokes' Theorem and the usual one, notice that

$$
\begin{aligned}
d(P d x+Q d y) & =\left(\frac{\partial Q}{\partial x}-\frac{\partial P}{\partial y}\right) d x d y \\
d(P d x+Q d y+R d z) & \\
& =\left(\frac{\partial R}{\partial y}-\frac{\partial Q}{\partial z}\right) d y d z+\left(\frac{\partial P}{\partial z}-\frac{\partial R}{\partial x}\right) d z d x \\
+ & \left(\frac{\partial Q}{\partial x}-\frac{\partial P}{\partial y}\right) d x d y .
\end{aligned}
$$

4. Fibre bundles. A simple example of a fibre bundle is the manifold formed by all the nonzero vectors tangent to a sphere of threedimensional Euclidean space. It is a topological manifold, but a very special one. However, it turns out that manifolds with similar properties play an important rôle in the application of topology to differential geometry. In the example of vectors tangent to a sphere the following three facts deserve attention:

(1) The spaces formed by the vectors with the same origin are homeomorphic to each other, and therefore to a fixed space which we call $F_{0}$.

(2) The tangent vectors drawn at all points of a neighborhood $U$ 
are homeomorphic to a topological product of $U$ and $F_{0}$. In particular, if $P \in U$, there is a homeomorphism $T$ which carries the tangent vectors with the origin $P$ to $F_{0}, T$ depending on $P$ and $U$.

(3) In the notation of (2) let us write $T(P, U)$ for $T$. If $P$ belongs to a second neighborhood $V$, the mapping $T(P, V) T^{-1}(P, U)$ is a homeomorphism of $F_{0}$ onto itself. It is possible to show that $T$ can be chosen so that $T(P, V) T^{-1}(P, U) \in G$, where $G$ is either the rotation group or the affine group in $F_{0}$.

The last statement can be demonstrated as follows: Let the neighborhoods be coordinate neighborhoods, such that $U$ is the set of all points whose local coordinates $u^{1}, u^{2}$ satisfy the inequalities $\left|u^{i}\right|<\epsilon, i=1,2$. With respect to the local coordinate system $u^{1}, u^{2}$ a vector $\mathfrak{x}$ has the components $X^{1}, X^{2}$. The vectors with origins in $U$ are thus decomposed into a topological product in an obvious way. If a point belongs to a neighborhood $V$ with the local coordinates $v^{1}, v^{2}$ and the vector $\mathfrak{x}$ has the components $Y^{1}, Y^{2}$ in the coordinate system $v^{1}, v^{2}$, then it is well known that

$$
Y^{1}=\frac{\partial v^{1}}{\partial u^{1}} X^{1}+\frac{\partial v^{1}}{\partial u^{2}} X^{2}, \quad Y^{2}=\frac{\partial v^{2}}{\partial u^{1}} X^{1}+\frac{\partial v^{2}}{\partial u^{2}} X^{2},
$$

which is an affine transformation.

Guided by this particular example, we shall give the definition of a general fibre bundle. For the terms and notation which will be used consistently later the following table of reference is given:

\begin{tabular}{l|l|l} 
General case & \multicolumn{1}{|c|}{ Special example } & Notation \\
\hline $\begin{array}{l}\text { Fibre bundle } \\
\text { Point of fibre } \\
\text { bundle }\end{array}$ & $\begin{array}{l}\text { Manifold of tangent vectors of a sphere } \\
\text { Tangent vector }\end{array}$ & $\mathfrak{F}$ \\
$\begin{array}{l}\text { Base space } \\
\text { Fibre }\end{array}$ & $\begin{array}{l}\text { Sphere } \\
\text { Projection }\end{array}$ & $\begin{array}{l}\text { Mapping of a tangent vector into its } \\
\text { origin }\end{array}$ \\
$\begin{array}{l}\text { Transformation } \\
\text { group in fibre or rotation group in space of } \\
\text { vectors with same origin }\end{array}$ & $G$ \\
\end{tabular}

We define a fibre bundle $\mathfrak{F}$ as a topological space which has the following properties:

(1) There exists a (continuous) mapping $\pi$ of $F$ onto another topological space $M: \pi(F)=M$. The space $M$ is called the base space and the mapping $\pi$ is called the projection. The complete inverse image 
$\pi^{-1}(p)$ of a point $p \in M$ is called the fibre at $p$. It will follow from assumptions made later that all the fibres are homeomorphic to each other and hence to a definite topological space $F_{0}$.

(2) There exists a family of neighborhoods which cover $M$. If $U$ is a neighborhood of the family, the inverse image $\pi^{-1}(U)$ is a topological product, which means that there exists a homeomorphism $\psi_{U}$, depending on $U$, such that

$$
\psi_{U}\left\{\pi^{-1}(U)\right\}=U \times F_{0},
$$

and that for every $p \in U$

$$
\psi_{U}\left\{\pi^{-1}(p)\right\}=p \times F_{0} .
$$

(3) Let $U, V$ be two such neighborhoods of $M$, and let $p \in U \cap V$. The mapping $\psi_{V}\left\{\psi_{U}^{-1}\left(p \times F_{0}\right)\right\}$ is a homeomorphism of $p \times F_{0}$, and hence of $F_{0}$, into itself. This homeomorphism belongs to a group $G$ given in advance in $F_{0}$.

For the cases in which we are interested both $M$ and $\mathfrak{F}$ will be supposed to be differentiable manifolds of class not less than 1 , and the group $G$ will be a Lie group.

In order to show the scope of the notion of a fibre bundle, the following further examples are given-all pertaining to a differentiable manifold $M$ of dimension $n$ and class not less than 1 :

(1) The tangent vectors of $M$ constitute a fibre bundle with $M$ as the base space.

(2) Let $\mathfrak{e}_{1}, \cdots, \mathfrak{e}_{p}, 1 \leqq p \leqq n$, be tangent vectors of $M$ with the common origin $P$. The elements $\left(P ; \mathrm{e}_{1}, \cdots, \mathrm{e}_{p}\right)$ constitute a fibre bundle over $M$. Another fibre bundle is constituted by the elements satisfying the condition that $e_{1}, \cdots, e_{p}$ are linearly independent. These fibre bundles have been studied by Stiefel and Whitney [26, $33]^{3}$

(3) We call a scalar density of weight $k$ a geometrical being, which has a component in each local coordinate system and whose components $f$ and $f^{*}$ in the local coordinate systems $x^{i}$ and $x^{* i}$ are connected by the relation

$$
f^{*}=f \cdot J^{k},
$$

where

$$
J=\partial\left(x^{1}, \cdots, x^{n}\right) / \partial\left(x^{* 1}, \cdots, x^{* n}\right) .
$$

The scalar densities or the nonzero scalar densities in $M$ constitute a fibre bundle over $M$.

\footnotetext{
${ }^{3}$ Numbers in brackets refer to the Bibliography at the end of the paper.
} 
(4) A general procedure of defining an important class of fibre bundles is as follows: Let $M$ be imbedded in a Euclidean space $E^{n+N}$ of dimension $n+N$. At a fixed point $O$ of $E^{n+N}$ let $H(n, N)$ be the Grassmann manifold formed by all the oriented linear subspaces of dimension $n$ through $O$. A mapping $T(M) \subset H(n, N)$ defines a fibre bundle over $M$ such that the fibre at each point $P$ of $M$ is the $n$-dimensional linear subspace through $P$ parallel to $T(P)$.

One of the important results in the theory of fibre bundles is the introduction of the so-called characteristic cocycle. To give its definition we assume $M$ to be a polyhedron, and let $K$ be a simplicial decomposition of $M$. Let $K^{r}$ be the $r$-dimensional skeleton of $K$, that is, the subcomplex of $K$ consisting of all simplexes of dimension not greater than $r$. We suppose the fibre bundle to be orientable in the sense explained by Steenrod [24]. Let $F_{0}$ be connected and have an abelian fundamental group. Under these assumptions the following theorems can be established:

THEOREM 4.1. Let $H^{i}\left(F_{0}\right)$ be the ith homology group (with integral efficients) of $F_{0}$. If

$$
H^{1}\left(F_{0}\right)=\cdots=H^{r-1}\left(F_{0}\right)=0,
$$

it is possible to define a continuous mapping $\phi$ of $K^{r}$ into $\mathfrak{F}$ such that $\pi \phi(p)=p, p \in K^{r}$.

THEOREM 4.2. With the same notation as in Theorem 4.1, if

$$
H^{1}\left(F_{0}\right)=\cdots=H^{r-1}\left(F_{0}\right)=0, \quad H^{r}\left(F_{0}\right) \neq 0,
$$

there exists a cohomology class (with $H^{r}\left(F_{0}\right)$ as the coefficient group) $\gamma^{r+1}$ of dimension $r+1$, whose vanishing is a necessary and sufficient condition that a continuous mapping $\phi$ of $K^{r+1}$ into $\mathfrak{F}$ can be defined such that $\pi \phi(p)=p, p \in K^{r+1}$. This cohomology class is a topological invariant of $\mathfrak{F}$.

The cohomology class $\gamma^{r+1}$ is called the characteristic cohomology class and any one of its cocycles a characteristic cocycle. If $M$ is an orientable manifold, the dual of $\gamma^{r+1}$ is a homology class of dimension $n-r-1$ and is called the characteristic homology class. In particular, if $M$ is a differentiable manifold and $\mathfrak{F}$ the fibre bundle of nonzero tangent vectors of $M$, the characteristic cocycle $\gamma$ is of dimension $n$ and its value for the fundamental cycle of $M$ is the Euler-Poincare characteristic of $M$. In this way we see that the theory of characteristic class generalizes the classical theory of vector fields on a differentiable manifold. 
5. Riemannian geometry. In the preceding sections are sketched the preliminaries for the theory of a geometric being. As an illustration we shall apply the tools so established to Riemannian geometry, the "simplest" and most important among the geometric beings. It will be seen that the results are essentially those given by Cartan and their relations with the now classical treatment by tensor analysis will also be indicated; but we have put ourselves in a more general viewpoint, which will lead to further developments.

Let $M$ be a differentiable manifold of dimension $n$ and class not less than 4. In $M$ suppose a Riemannian metric be given, which, in terms of a local coordinate system $x^{i}$, is defined by a positive definite quadratic differential form ${ }^{4}$

$$
d s^{2}=\sum_{i, j} g_{i j}\left(x^{k}\right)\left(d x^{i} d x^{j}\right),
$$$$
g_{i j}=g_{j i}
$$

The manifold $M$ is then called a Riemannian manifold.

Consider a point $P$ of $M$ and the contravariant tangent vectors of $M$ having the origin $P$. With the differential form (16) (or the tensor $g_{i j}$ ) the scalar product of two vectors $e, f$ can be defined, which we denote by $e \cdot f$. A vector $e$ is called a unit vector if $e^{2}=1$. An ordered set of $n$ vectors $e_{1}, \cdots, e_{n}$ is said to constitute a frame (ennuple), if

$$
\mathrm{e}_{i} \mathrm{e}_{j}=\delta_{i j}=\left\{\begin{array}{ll}
1, & i=j, \\
0, & i \neq j,
\end{array} \quad i, j=1, \cdots, n .\right.
$$

A differentiable curve through $P$ is defined by the equations

$$
x^{i}=x^{i}(s),
$$

where $s$ is the arc length of the curve and the functions are of class not less than 1 . We denote by $d P / d s$ the unit tangent vector of the curve at $P$. It is easy to show, by referring to a local coordinate system, that there exists a vector (which we shall denote by $d P$ ) whose components with respect to a frame are linear differential forms and which is equal to the product of $d s$ and the unit tangent vector along a curve. This vector is intrinsic, that is, independent of the choice of coordinates. Referred to a frame $e_{1}, \cdots, e_{n}$, we can write

$$
d P=\omega_{1} e_{1}+\cdots+\omega_{n} e_{n},
$$

where $\omega_{1}, \cdots, \omega_{n}$ are linear differential forms. Then we have, along a curve, $(d P / d s)^{2}=1$ or

4 The differential form in question is an ordinary and not an exterior differential form. As a means of distinction, we insert a parenthesis about the differentials when the former is the case. 


$$
d s^{2}=\left(\omega_{1}\right)^{2}+\cdots+\left(\omega_{n}\right)^{2} .
$$

The forms $\omega_{i}, i=1, \cdots, n$, are therefore the forms the sum of whose squares is equal to the given (ordinary) quadratic differential form.

As the origin $P$ runs over $M$, the frames $\mathrm{e}_{1}, \cdots, \mathrm{e}_{n}$ constitute a fibre bundle with $M$ as the base space. Each fibre is topologically the space of all frames having the same origin. The fibre bundle is of dimension $n(n+1) / 2$ and each fibre is of dimension $n(n-1) / 2$. The group which operates in each fibre is the group of orthogonal transformations.

There is yet no intrinsic meaning attached to the vectors $d e_{i}$. We want to see whether it is possible to define intrinsically certain differential forms $\omega_{i j}$ such that

$$
d e_{i}=\sum_{j} \omega_{i j} e_{j}
$$

From (17) it follows that $\omega_{i j}$ has to be skew-symmetric in its indices:

$$
\omega_{i j}+\omega_{j i}=0 \text {. }
$$

The answer to our question is given by the following theorem:

THEOREM 5.1. There exists one, and only one, set of linear differential forms $\omega_{i j}$ in the fibre bundle such that the equation (21) and the equation

$$
d(d P)=0
$$

hold.

In fact, the condition (23) gives, when expanded,

$$
d \omega_{i}-\sum_{j} \omega_{j} \omega_{j i}=0 .
$$

To calculate $d \omega_{i}$ we make use of a local coordinate system $x^{i}$ and take a definite decomposition of $d s^{2}$ as a sum of squares:

$$
d s^{2}=\left(\theta_{1}\right)^{2}+\cdots+\left(\theta_{n}\right)^{2},
$$

where

$$
\theta_{i}=\sum_{i} a_{i j}(x) d x^{j}
$$

Since (20) and (25) give two decompositions of the same ordinary quadratic differential form as sums of squares, we have

$$
\omega_{i}=\sum u_{i j} \theta_{j},
$$

where $u_{i j}$ are the elements of an orthogonal matrix: 


$$
U=\left(u_{i j}\right), \quad U U^{\prime}=I=\text { identity matrix. }
$$

Forming the exterior derivative of the equation (26), we get

$$
d \omega_{i}=\sum d u_{i j} \theta_{j}+\sum u_{i j} d \theta_{j},
$$

which shows that $d \omega_{i}$ is of the form

$$
d \omega_{i}=\sum \omega_{j} \phi_{j i},
$$

where $\phi_{i i}$ are linear differential forms in the variables $x^{i}, u_{i j}$ and can be explicitly calculated. Substitution into (24) gives

$$
\sum \omega_{j}\left(\omega_{j i}-\phi_{j i}\right)=0,
$$

which gives in turn

$$
\omega_{j i}-\phi_{j i}=\sum \lambda_{j i k} \omega_{k}, \quad \quad \lambda_{j i k}=\lambda_{k i j \cdot}
$$

In this equation the forms $\phi_{j i}$ are known and the quantities $\lambda_{j i k}$ will be determined in order that $\omega_{j i}$ be determined. Since $\omega_{i j}$ is skew-symmetric in its indices, we have

$$
-\left(\phi_{i j}+\phi_{j i}\right)=\sum\left(\lambda_{i j k}+\lambda_{j i k}\right) \omega_{k} .
$$

This equation shows that $\phi_{i j}+\phi_{j i}$ is of the form

$$
\phi_{i j}+\phi_{j i}=-\sum A_{i j k} \omega_{k}, \quad A_{i j k}=A_{j i k},
$$

and that then

$$
\lambda_{i j k}+\lambda_{j i k}=A_{i j k} .
$$

The quantities $\lambda_{i j k}$, being symmetric in the first and third indices, are then uniquely determined and are given by

$$
\lambda_{i j k}=\left(-A_{i k j}+A_{j k i}+A_{i j k}\right) / 2 .
$$

This proves our theorem.

The equation (21) may be interpreted as giving a means of transporting a vector at an infinitesimally near point of $P$ to a vector at $P$. It is essentially the notion known as the parallelism of Levi-Civita.

By forming the exterior derivative of (24) and making use of these equations themselves, we get

$$
\sum_{j} \omega_{j}\left(\sum_{k} \omega_{i k} \omega_{k j}-d \omega_{i j}\right)=0 .
$$

It follows easily from this that we can write

$$
d \omega_{i j}=\sum_{k} \omega_{i k} \omega_{k j}+\Omega_{i j}
$$


where $\Omega_{i j}$ are of the form

$$
\begin{gathered}
\Omega_{i j}=\sum R_{i j, k l} \omega_{k} \omega_{l}, \quad R_{i j, k l}+R_{i j, l k}=0, \\
\Omega_{i j}+\Omega_{j i}=0 .
\end{gathered}
$$

We shall call the forms $\Omega_{i j}$ the curvature forms.

In a slightly different version our results may be summarized in the theorem:

TheOREM 5.2. Let $M$ be a Riemannian manifold of dimension $n$ and let $\mathfrak{F}$ be the fibre bundle of all frames of $M$. There is a unique way to define in $\mathfrak{F}$ (of dimension $n(n+1) / 2$ ) a set of $n(n+1) / 2$ linearly independent linear differential forms $\omega_{i}, \omega_{i j}$, which satisfy the equations (19), (21), (24).

Let us indicate briefly the relations of these considerations to the ordinary treatment by means of tensor analysis. In tensor analysis emphasis is laid particularly on the local coordinate system. The procedure is as follows: With respect to the local coordinates $x^{i}$ let $\mathrm{f}_{k}$ be the velocity vector of the coordinate curve $x^{i}=$ const., $i \neq k$, with $x^{k}$ as the time. Then we have

$$
\mathfrak{f}_{i} \cdot \mathfrak{f}_{k}=g_{i k},
$$

and

$$
d P=d x^{1} \cdot f_{1}+\cdots+d x^{n} \cdot f_{n} .
$$

The components $X^{i}$ of a vector $\mathfrak{x}$ with respect to the vectors $\mathfrak{f}_{k}$ are defined by the equation

$$
\mathfrak{x}=\sum X^{i} \mathfrak{f}_{i} \text {. }
$$

By an argument analogous to the proof of Theorem 5.1 it can be shown that there exists a set of linear differential forms such that

$$
d f_{i}=\sum \theta_{i}^{j} f_{j}
$$

and

$$
d(d P)=0 \text {. }
$$

The vector $\mathfrak{x}+d \mathfrak{x}$ is called parallel to $\mathfrak{x}$ if $d \mathfrak{x}=0$, which can be written, by using equations (36) and (37),

$$
d X^{i}+\sum \theta_{j}^{i} X^{j}=0 \text {. }
$$

These equations are easily recognized to be the well known equations which define the parallelism of Levi-Civita. 
The important fact in Riemannian geometry is the necessity of taking into consideration not only the Riemannian manifold itself but the fibre bundle over it. In our approach we consider directly the fibre bundle. The usual tensor approach avoids it by laying emphasis on the local coordinate system. Quantities of geometrical interest are those which obey a simple transformation law under changes of the local coordinate systems. For Riemannian geometry the two methods are essentially the same, so far as local problems are concerned. It is, however, to be remarked that even for local problems their generalizations to other geometric beings will lead to different formulations. We shall give in the next section a systematic way of treating the local theory of a geometric being.

6. Method of equivalence. So far the introduction given in the above section of the fibre bundle over a Riemannian manifold $M$ seems to be accidental. The underlying reason will be most clear, if we start with the following problem:

Let two Riemannian metrics be given by the positive definite quadratic differential forms

$$
\begin{aligned}
d s^{2} & =\sum_{i, j} g_{i j}(x)\left(d x^{i} d x^{j}\right), & g_{i j} & =g_{j i}, \\
d s^{* 2} & =\sum_{i, j} g_{i j}{ }^{*}\left(x^{*}\right)\left(d x^{* i} d x^{* j}\right), & g_{i j}{ }^{*} & =g_{j i}{ }^{*},
\end{aligned}
$$

in two coordinate neighborhoods $U, U^{*}$ respectively. We determine the conditions that a neighborhood $V \subset U$ can be mapped by a differentiable mapping (of sufficiently large class) with nonvanishing functional determinant into $U^{*}$ such that

$$
d s^{* 2}=d s^{2} .
$$

This so-called form problem was solved by Christoffel and Lipschitz. It is clear that if the problem has a solution, the local theories of the two Riemannian metrics are essentially the same.

To give a different formulation of our problem we determine two sets of linear differential forms $\theta_{i}(x, d x)$ and $\theta_{i}^{*}\left(x^{*}, d x^{*}\right)$ such that

$$
d s^{2}=\left(\theta_{1}\right)^{2}+\cdots+\left(\theta_{n}\right)^{2}, \quad d s^{* 2}=\left(\theta_{1}^{*}\right)^{2}+\cdots+\left(\theta_{n}^{*}\right)^{2} .
$$

Our problem will have a solution if and only if the differentiable mapping in question and the functions $u_{i j}(x)$ can be determined in a neighborhood $V \subset U$ such that

$$
\theta_{i}^{*}\left(x^{*}, d x^{*}\right)=\sum_{j} u_{i j}(x) \theta_{j}(x, d x)
$$

where the matrix 


$$
U=\left(u_{i j}\right)
$$

is an orthogonal matrix.

In this way our problem, originally formulated in terms of quadratic differential forms, is reduced to one in linear differential forms. In this formulation the problem is a particular case of a more general problem, which was first stated and solved by Elie Cartan. Cartan's problem is as follows:

Let $\theta_{i}(x, d x)$ and $\theta_{i}^{*}\left(x^{*}, d x^{*}\right)$ be two sets of $n$ linearly independent linear differential forms in the coordinate neighborhoods $U, U^{*}$ of the coordinates $x^{i}, x^{* i}, i=1, \cdots, n$. Let $\Gamma$ be a linear group in the vector space of $n$ dimensions. We wish to determine the conditions that there exists a differentiable mapping with a nonvanishing functional determinant of a neighborhood $V \subset U$ into $U^{*}$ such that

$$
\theta_{i}^{*}=\sum_{j} u_{i j}(x) \theta_{j}
$$

where the linear transformation belongs to the given linear group $\Gamma$.

In our case $\Gamma$ is the orthogonal group.

The discussion of this local problem requires Cartan's theory of Pfaffian systems in involution and is not simple. For details we refer to $[3]$.

We only remark that if $\Gamma$ consists of the identity only, the solution of this problem is relatively simple. The general procedure is to reduce the problem to this particular case by the introduction of new variables. We shall illustrate this method by considering our form problem. Let $u_{i j}$ and $u_{i j}{ }^{*}$ be two sets of new variables which are elements of the orthogonal matrices

$$
U=\left(u_{i j}\right), \quad U^{*}=\left(u_{i j}^{*}\right),
$$

and let us put

$$
\omega_{i}=\sum u_{i j} \theta_{j}, \quad \omega_{i}^{*}=\sum u_{i j}^{*} \theta_{j}^{*} .
$$

Our form problem will have a solution if and only if there exists a differentiable mapping of a neighborhood $V \subset U$ into $U^{*}$ and an orthogonal matrix $X$, whose elements are functions in $V$, such that under this mapping and the mapping between $\left(u_{i j}\right)$ and $\left(u_{i j}{ }^{*}\right)$ defined by

$$
U^{*} X=U,
$$

we have

$$
\omega_{i}^{*}=\omega_{i} .
$$


From the last equation we derive

$$
d \omega_{i}^{*}=d \omega_{i} .
$$

By an argument exactly identical to one used in the last section it can be shown that there exists one and only one set of linear differential forms $\omega_{i j}$ in $x^{i}, u_{i j}$, which are skew-symmetric in their indices and which satisfy the equations

$$
d \omega_{i}=\sum \omega_{j} \omega_{j i} .
$$

Similarly, we find a set $\omega_{i j}{ }^{*}\left(=-\omega_{j i}{ }^{*}\right)$ of linear differential forms such that

$$
d \omega_{i}^{*}=\sum \omega_{j}^{*} \omega_{j i}^{*} .
$$

When (46) and (47) hold, it is easy to derive

$$
\omega_{i j}{ }^{*}=\omega_{i j} .
$$

Let $V$ be a sufficiently small neighborhood of $U$ and let $O(n)$ be the group manifold of the orthogonal group. We consider the topological product $V \times O(n)$. Similarly, we have the topological product $V^{*} \times O^{*}(n)$. In order that our form problem possess a solution it is necessary and sufficient that there exist a differentiable mapping between $V \times O(n)$ and $V^{*} \times O^{*}(n)$ such that under this mapping the equations (46) and (48) hold. It is easy to see that the forms $\omega_{i}, \omega_{i j}$, whose number $n(n+1) / 2$ is equal to the dimension of $V \times O(n)$, are linearly independent. Thus our problem is reduced to Cartan's general problem with $\Gamma$ consisting of the identity only.

It can therefore be seen that the discussions of the last section are nothing else than a geometrical treatment of the above analytic considerations. We summarize these results as follows:

Given a Riemannian manifold $M$ of dimension $n$ with a positive definite quadratic differential form $d s^{2}$. To $M$ is associated the fibre bundle, with $M$ as its base space, of all sets of linear differential forms (that is, covariant vectors) the sum of whose squares is equal to $d s^{2}$. The fibre bundle is of dimension $n(n+1) / 2$ and there exists in it a set of $n(n+1) / 2$ linearly independent linear differential forms $\omega_{i}, \omega_{i j}$ which are invariant. There exists locally a differentiable mapping which carries one $d s^{2}$ into another when and only when such a mapping exists in the corresponding fibre bundles under which the forms $\omega_{i}, \omega_{i j}$ are respectively equal.

We may say that the deep reason that this very fibre bundle (namely, the one whose fibres are topologically the manifolds $O(n)$ ) is attached to a Riemannian manifold comes as a result of the solution 
of the form problem. From the case of the Riemannian manifold it is clear that, for the theory of other kinds of geometric beings, the solution of a corresponding local "equivalence problem" is a natural preliminary step for the establishment of a proper theory in the large. In fact, from the solution of the equivalence problem the nature of the fibre bundle to be attached to the manifold can be determined.

There is a great variety of geometric beings which are of interest in differential geometry. In order to illustrate our method we shall take as a further example the so-called geometry of paths. Let $M$ be a differentiable manifold of dimension $n$. In a neighborhood with the local coordinates $x^{i}$ let there be given a system of differential equations of the second order:

$$
\frac{d^{2} x^{i}}{d t^{2}}+\sum \Gamma_{j k}^{i}(x) \frac{d x^{i}}{d t} \frac{d x^{k}}{d t}=F(x, t) \frac{d x^{i}}{d t},
$$

where the functions $\Gamma_{j k}^{f}, F$ are supposed to be differentiable of class not less than 3 . The integral curves of the differential system (49) are called the paths. They have the property that through every point of $M$ and tangent to every vector through that point there passes one and only one path. The independent variable $t$ is a parameter on the path. It is irrelevant in the sense that a change of parameter $t=\phi(\tau)$, where $\phi(\tau)$ is differentiable, gives the same path. In short, our manifold $M$ carries a system of paths which is defined in each local coordinate system by a differential system of the form (49) and our problem is to study the geometrical properties in $M$ arising from the paths. An important particular case of this problem is the case that $M$ is the projective space of $n$ dimensions and the paths are the straight lines in $M$, which, in a suitable local coordinate system, are defined by the system (49), with $\Gamma_{j k}^{f}=0$.

Following the spirit of our solution of the form problem we start by considering the following problem of equivalence: In another coordinate neighborhood with the local coordinates $x^{* i}$ let a system of paths be given by a differential system analogous to (49). Determine the conditions under which a differentiable mapping between the coordinates $x^{i}$ and $x^{* i}$, which carries one system of paths into another, exists.

To apply the method of equivalence we shall write the system (49) in a system of total differential equations. For simplicity let us suppose that, for the integral curves of (49) under consideration, $x^{n}$ is not a constant. We can then take $x^{n}$ to be the parameter along the curves, and the system (49) can be written 


$$
\frac{d x^{\alpha}}{d x^{n}}=y^{\alpha}, \quad \frac{d y^{\alpha}}{d x^{n}}+\sum \Gamma_{j k}^{\alpha} y^{j} y^{k}=F y^{\alpha}, \quad \sum \Gamma_{j k}^{n} y^{j} y^{k}=F,
$$

where the index $\alpha$ runs from 1 to $n-1$ and $y^{n}=1$. This system can be written as a system of total differential equations as follows:

$$
\begin{aligned}
\theta^{\alpha} & =d x^{\alpha}-y^{\alpha} d x^{n}=0, \\
\pi^{\alpha} & =d y^{\alpha}+\left\{\sum \Gamma_{j k}^{\alpha} y^{j} y^{k}-y^{\alpha} \sum \Gamma_{j k}^{n} y^{j} y^{k}\right\} d x^{n}=0 .
\end{aligned}
$$

We introduce also the form $d x^{n}$, so that $\theta^{\alpha}, d x^{n}$ constitute a set of linearly independent linear differential forms in the coordinates $x^{i}$. On the other manifold with the local coordinates $x^{* i}$ we shall introduce in a similar way the new variables $y^{* \alpha}$ and the forms $\theta^{* \alpha}, d x^{* n}$, $\pi^{* \alpha}$. Let us write

$$
\Theta=\left(\begin{array}{c}
d x^{n} \\
\theta^{\alpha} \\
\pi^{\alpha}
\end{array}\right), \quad \Theta^{*}=\left(\begin{array}{c}
d x^{* n} \\
\theta^{* \alpha} \\
\pi^{* \alpha}
\end{array}\right),
$$

and let us introduce the matrix

$$
U=\left(\begin{array}{ccc}
A(1,1) & B(1, n-1) & 0 \\
0 & C(n-1, n-1) & 0 \\
0 & D(n-1, n-1) & E(n-1, n-1)
\end{array}\right)
$$

whose nonzero elements are independent variables, and where the numbers in the parentheses denote the numbers of rows and columns of the sub-matrices in question. It is easy to show that our problem of equivalence possesses a solution when and only when there is a differentiable mapping between two neighborhoods $\left(x^{i}, y^{\alpha}\right)$ and $\left(x^{* i}, y^{* \alpha}\right)$ and the elements of $U$ can be determined as functions of $x^{i}, y^{\alpha}$, such that under this mapping the matrix equation

$$
\Theta^{*}=U \Theta
$$

holds. Now in the linear vector space of $2 n-1$ dimensions all the matrices $U$ form a group. Hence in this formulation our problem of equivalence is a particular case of the problem of equivalence of Cartan stated above.

The problem can be solved by applying Cartan's general procedure. We shall not give the details here. The solution of a problem which contains this problem as a particular case was given in [12].

Our final result can be stated in the theorem:

THEOREM 6.1. In a differentiable manifold $M$ of dimension $n$ and 
class not less than 3 let a system of paths be given, defined locally by the differential equations (49). It is possible to introduce in a neighborhood of $M$ with the local coordinates $x^{i}, n(n+1)$ other variables $u_{A}$, $A=1, \cdots, n(n+1)$, and to define $n(n+2)$ linearly independent linear differential forms $\omega_{B}, B=1, \cdots, n(n+2)$, in the variables $x^{i}, u_{A}$, having the following property: $A$ necessary and sufficient condition that a local differentiable mappings exists, which carries one system of paths into another with the local coordinates $x^{* i}$ and the new variables $u_{A}{ }^{*}$, and so on, is that there exists a differentiable mapping between two neighborhoods of the spaces $\left(x^{i}, u_{A}\right)$ and $\left(x^{* i}, u_{A}^{*}\right)$ under which the forms $\omega_{B}$ and $\omega_{B}{ }^{*}$ are mapped into each other.

With respect to a local coordinate system $x^{i}$ the variables $x^{i}, u_{A}$, $A=1, \cdots, n(n+1)$, determine a topological product. We are thus led to consider the fibre bundle over $M$, which is of dimension $n(n+2)$ and is locally given by the coordinates $x^{i}, u_{A}$. In the fibre bundle there are defined exactly $n(n+2)$ intrinsic linearly independent linear differential forms. The significance of this fibre bundle to the differential geometry of paths is evident. It is important to point out that in the geometry of paths it is, in view of our Theorem 6.1, this fibre bundle, and not the fibre bundle of tangent vectors of $M$, which plays the fundamental rôle.

From the above discussions of Riemannian geometry and the geometry of paths it can be seen that the method of equivalence offers the best weapon for a frontal attack on the problem of a geometric being. Only from the outcome of the solution of the problem of equivalence can it be decided the most important kind of fibre bundle to be defined over the manifold. It is of course possible to define, in a more or less intuitive way, other kinds of fibre bundles over the manifold, but they will play a minor rôle. We wish also to remark that the solution of this local problem of equivalence is not always simple. So far as the writer is aware, the problem of equivalence for a skew-symmetric covariant tensor of order two, for instance, has not been explicitly solved (although the solution is theoretically always possible).

7. Relations between the fibre bundle and its base manifold. We have attempted to show in the above, in the cases of Riemannian geometry and the geometry of paths, how the local theory of a geometric being leads naturally to the fibre bundle to be associated to the manifold on which the geometric being is defined. The fact remains true of any geometric being, but its proof requires the fundamental theorem on partial differential equations to the effect that every system of partial differential equations can be "prolonged" to 
a system in involution, and we shall not enter into its discussion. It is to be remarked that for problems of this sort, as for any problem concerned with both pure and applied mathematics, the general result and the particular cases offer entirely different problems, one being an existence theorem and the other being an explicit solution. In our examples we solve the problems explicitly, thereby insuring the existence of the solution.

After defining over the given manifold $M$ a fibre bundle $\mathfrak{F}$, the next step is to study their relationship. Before proceeding farther, let us remark that the fibre bundle $\mathfrak{F}$ will give rise to new fibre bundles over $M$ by identification. Consider, for instance, the case that $M$ is a Riemannian manifold. Let $P e_{1} \cdots e_{n}$ be a frame in $M$. For any integer $p, 1 \leqq p \leqq n$, we shall define a class of frames to be all frames $P e_{1} \cdots e_{n}$ such that the point $P$ and the vectors $e_{1}, \cdots, e_{p}$ are identical. These classes of frames constitute, with a natural topology, a fibre bundle over $M$, which we denote by $\mathfrak{F}^{(p)} \cdot \mathfrak{F}^{(p)}$ is the fibre bundle of all ordered sets of $p$ mutually perpendicular unit vectors of $M$. In particular, $\mathfrak{F}^{(1)}$ is the fibre bundle of unit vectors of $M$ and $\mathfrak{F}^{(n)}$ is the fibre bundle $\mathfrak{F}$ itself. For distinction we shall call $\mathfrak{F}$ the principal fibre bundle of $M$ and all others obtained by identification the associated fibre bundles. We shall consistently assume that the identification is made only in each fibre, so that the projection of the associated bundle onto the base manifold is the one induced by that of the principal bundle. It is seen that this process is very general.

Let $\mathfrak{F}$ be the principal fibre bundle over $M$ and $\$$ an associated fibre bundle over $M$. There is a projection $\pi(\mathfrak{F}) \subset M$ and a projection $\pi_{1}(\Im) \subset M$, and also a projection $\pi_{2}(\mathfrak{F}) \subset(B)$, defined by assigning to a point of $\mathfrak{F}$ the class to which it belongs during the identification. From the remark made at the end of the last paragraph it is clear that $\pi=\pi_{1} \pi_{2}$. We also remember that a set of intrinsic linearly independent linear differential forms is defined in $\mathfrak{F}$ by the geometric being, whose number is equal to the dimension of $\mathfrak{F}$. Our main aim will be to see how these differential forms behave under the projections $\pi, \pi_{1}, \pi_{2}$.

In order to understand this relationship more clearly we shall make a digression to recall some elementary facts in combinatorial topology. Let $K$ be a finite complex of dimension $n$, whose simplexes we denote by $\sigma_{\imath}^{r}, 0 \leqq r \leqq n, 1 \leqq i \leqq \alpha_{r}$. Let $R$ be a commutative ring. A chain of dimension $r$ or an $r$-chain is a sum

$$
C^{r}=\sum_{i=1}^{\alpha_{r}} \lambda_{i} \sigma_{i}^{*}, \quad \lambda_{i} \in R .
$$

To the chains $C^{r}$ we introduce a boundary operator $\partial$, which is linear: 


$$
\partial\left(C_{1}^{r}+C_{2}^{r}\right)=\partial C_{1}^{r}+\partial C_{2}^{r}, \quad \partial\left(\lambda C^{r}\right)=\lambda \partial C^{r}, \quad \lambda \in R,
$$

and is defined for the simplexes $\sigma_{\imath}^{r}$ by the so-called incidence relations:

$$
\partial \sigma_{i}^{r}=\sum_{j=1}^{\alpha_{r-1}} \eta_{i j}^{(r)} \sigma_{j}^{r-1}
$$

The relations (55) and (56) completely determine the boundary operator $\partial$ and the chain $\partial C^{r}$ of dimension $r-1$ is called the boundary of $C^{r}$. It is easy to verify that

$$
\partial \partial C^{r}=0 .
$$

A chain whose boundary is zero is called a cycle. Equation (57) shows that a chain which is the boundary of another chain is a cycle, called a bounding cycle. The cycles form an abelian group and the bounding cycles a subgroup of it. Their quotient group is called the $r$-dimensional homology group of $K$ (with the additive group of $R$ as its coefficient group).

A cochain $\gamma^{r}$ of dimension $r$ is a linear function of the $r$-chains. We shall denote the function by the notation $\gamma^{r} \cdot C^{r}$, which has therefore the properties:

$$
\gamma^{r} \cdot\left(C_{1}^{r}+C_{2}^{r}\right)=\gamma^{r} \cdot C_{1}^{r}+\gamma^{r} \cdot C_{2}^{r}, \quad \gamma^{r} \cdot\left(\lambda C^{r}\right)=\lambda \gamma^{r} \cdot C^{r}, \quad \lambda \in R .
$$

From a cochain $\gamma^{r}$ of dimension $r$ we define a cochain $\delta \gamma^{r}$ of dimension $r+1$, called its coboundary, by means of the relation

$$
\delta \gamma^{r} \cdot C^{r+1}=\gamma^{r} \cdot\left(\partial C^{r+1}\right) .
$$

It then follows from (57) and (59) that

$$
\delta \delta \gamma^{r}=0 .
$$

A cochain whose coboundary is zero is called a cocycle. All the cocycles of dimension $r$ form an abelian group, which contains as subgroup the group of all cocycles which are coboundaries. Their quotient group is called the $r$-dimensional cohomology group of $K$.

Now, let $M$ be a differentiable manifold of dimension $n$ and class not less than 2. Let $\omega$ be a differential form of degree $r$ in $M$, whose coefficients are of class not less than 2 . As we have remarked in $\$ 3$, it is possible to define in a rigorous manner the integral of $\omega$ over an $r$-simplex and also the integral of $\omega$ over an $r$-chain. Moreover, the functional $\int_{C^{r} \omega}$ is linear in the $r$-chains $C^{r}$. Hence it defines an $r$-cochain with the ring of real numbers as the coefficient ring. When there is no confusion, we shall speak of $\omega$ as an $r$-cochain.

From (13) the Theorem of Stokes can be written in the form 


$$
\int_{C^{r+1}} d \omega=\int_{\partial C^{r+1}} \omega .
$$

Comparison with (59) shows that if $\omega$ defines an $r$-cochain, then $d \omega$ defines its coboundary. It follows that the $r$-cochain $\omega$ is a cocycle if and only if $d \omega=0$ (in which case $\omega$ is called exact), and that $\omega$ is a coboundary if there exists a form $\theta$ whose exterior derivative $d \theta$ is equal to $\omega$ (in which case $\omega$ is called derived).

It was de Rham [22] who proved the important theorem that to every $r$-cochain $\gamma$ (with real or rational coefficient group) there exists a differential form $\omega$ of degree $r$ which defines $\gamma$ according to the above process. We see from $\$ 4$ that from the fibre bundles over $M$ there are the characteristic cocycles in $M$ which are invariants of the fibre bundles. A theorem in the differential geometry in the large will be obtained, whenever it is possible to define a characteristic cocycle by means of a differential form constructed locally from the geometric being.

Suppose now that a simplicial mapping $f$ exists, which maps a complex $K$ into a complex $K^{*}$. The mapping $f$ induces a mapping $\phi$ of the chains of $K$ into the chains of $K^{*}$. From $\phi$ a mapping $\psi$ of the cochains of $K^{*}$ into the cochains of $K$ can be defined. In fact, if $\gamma^{*}$ is a cochain of dimension $r$ of $K^{*}$, we define $\psi \gamma^{*} \cdot \sigma^{r}=\gamma^{*} \cdot \phi \sigma^{r}$ for every simplex $\sigma^{r}$ of $K$. This so-called inverse mapping $\psi$ maps cocycles of $K^{*}$ into cocycles of $K$ and cocycles of the same cohomology class into cocycles of the same cohomology class. Therefore it induces a mapping of each cohomology group of $K^{*}$ into the cohomology group of the same dimension of $K$ and it can be proved that it is a homomorphism. It can also be proved that the homomorphism remains unchanged, if $f$ is replaced by a mapping homotopic to $f$. The result is that we can define from a continuous mapping of a polyhedron $P$ into a polyhedron $P^{*}$ a homomorphism of a cohomology group of $P^{*}$ into one of the same dimension of $P$.

In the case of a fibre bundle $\mathfrak{F}$ over $M$ in which we are interested, there is a continuous mapping of $\mathfrak{F}$ into $M$, namely the projection. We assume that both $\mathfrak{F}$ and $M$ are differentiable manifolds. According to the above, the projection induces a mapping of a cohomology group of $M$ into one of the same dimension of $\mathfrak{F}$. In particular, an exact differential form of $M$ will be mapped into an exact differential form of $\mathfrak{F}$.

Consider the case that $M$ is a differentiable manifold in which a geometric being is given. Let $\mathfrak{F}$ be the principal fibre bundle and $(5)$ an associated fibre bundle over $M$. It is known that there is a projection $\pi_{2}(\mathfrak{F}) \subset(\mathbb{S}$. There are also linear differential forms defined in $\mathfrak{F}$ 
by the geometric being in $M$. From the linear differential forms it is possible to construct, by operations of the Grassmann algebra and the Cartan calculus, differential forms of higher degree. For any such differential form it is important to decide whether it is the image of a differential form of $\&$ under the inverse mapping induced by $\pi_{\mathbf{2}}$. There are several criterions for this problem. We shall give a criterion for the case that $M$ is a Riemannian manifold, but the process can be readily generalized to other geometric beings.

Let $\mathbb{S}=F^{(p)}$ and let the forms $\omega_{i}, \omega_{i j}, \Omega_{i j}$ be defined in the principal fibre bundle $\mathfrak{F}$. Let II be constructed from $\omega_{i}, \omega_{i j}, \Omega_{i j}$ by operations of the Grassmann algebra. We wish to decide whether $\Pi$ is the image of a form in $\$$.

For this purpose let us use in this paragraph the following ranges of indices:

$$
1 \leqq \alpha, \beta \leqq p, \quad p+1 \leqq r, s \leqq n, \quad 1 \leqq i, k \leqq n .
$$

Let $\delta$ be an operation (not coboundary) under which the figure $P \mathrm{e}_{1} \cdots \mathrm{e}_{p}$ remains unchanged, so that

$$
\omega_{i}(\delta)=0, \quad \omega_{\alpha i}(\delta)=0 .
$$

We also put

$$
\omega_{r s}(\delta)=e_{r s}
$$

From (24) and (32) we have

$$
\begin{aligned}
\delta \omega_{\alpha} & =0, \quad \delta \omega_{r}=\sum e_{r s} \omega_{s,}, \delta \omega_{\alpha \beta}=0, \quad \delta \omega_{\alpha r}=-\sum e_{s r} \omega_{\alpha s,} \\
\delta \Omega_{\alpha \beta} & =0, \quad \delta \Omega_{\alpha r}=\sum e_{r s} \Omega_{\alpha s,}, \quad \delta \Omega_{r s}=\sum\left(e_{r t} \Omega_{t s}-\Omega_{r t} e_{t s}\right) .
\end{aligned}
$$

Then we have the following theorem:

THEOREM 7.1. A form $\Pi$ is the image of a differential form in $\mathfrak{F}^{(p)}$ if and only if $\delta \Pi=0$.

This theorem is also true for $p=0$, if we make the convention $\mathfrak{F}^{(0)}=M$.

If a form in $\mathfrak{F}$ is the image of a form in $\mathfrak{F}^{(p)}$, we shall say simply that it is a form of $\mathfrak{F}^{(p)}$.

It can easily be verified, by means of our criterion, that the following forms are forms of $M$ :

$$
\begin{aligned}
\Delta_{m} & =\sum \Omega_{i_{1} i_{2}} \Omega_{i_{2} i_{3}} \cdots \Omega_{i_{2 m} i_{1}}, \quad 1 \leqq m \leqq n / 4, \\
\Delta_{0} & =\sum \epsilon_{i_{1}} \cdots i_{n} \Omega_{i_{1} i_{2}} \cdots \Omega_{i_{n-1} i_{n}}, \quad \text { if } n \text { is even, }
\end{aligned}
$$

where $\epsilon_{i_{1}} \ldots i_{n}$ is the Kronecker index, which is +1 or -1 , according 
as $i_{1}, \cdots, i_{n}$ forms an even or odd permutation of $1, \cdots, n$, and is otherwise zero.

Important is the case of an exact form of $M$ which is not derived in $M$, but has an image in a fibre bundle which is derived. The form $\Delta_{0}$ in (64) has, for instance, an image in $F^{(1)}$, which is derived. It is on this fact that the generalized Gauss-Bonnet formula was proved in a simple way [10]. A further example to illustrate this idea will be given for Riemannian manifolds of four dimensions. (Compare [14].)

8. Theorem of Pontrjagin. From a topological viewpoint Pontrjagin has in two recent notes $[20,21]$ studied a problem related to ours. We shall summarize here his results in a slightly more general version which will allow its generalization to complex analytic Hermitian manifolds.

Let $E^{n+N}$ be a real oriented Euclidean space of dimension $n+N$. In $E^{n+N}$ we consider the Grassmann manifold $H(n, N)$, formed by all the oriented $n$-dimensional linear spaces through the origin. To each submanifold $M^{\prime}$ of $H(n, N)$ there is defined in a natural way a sphere bundle with $M^{\prime}$ as the base space, that is, a fibre bundle whose fibres consist of spheres cut on the unit hypersphere about the origin of $E^{n+N}$ by the $n$-dimensional linear subspaces of $M^{\prime}$. Given any manifold $M$, and a mapping $f(M) \subset H(n, N)$, a sphere bundle can be defined over $M$ as base space by taking as the fibre attached to a point $P$ of $M$ the unit hypersphere in $f(P)$. With a proper definition of equivalence of sphere bundles, Whitney and Steenrod have proved the following theorem $[25,33]$ :

THEOREM 8.1. To a given bundle of spheres of dimension $n-1$ over a compact manifold $M$ there exists a mapping $f(M) \subset H(n, N)$, which defines a sphere bundle over $M$ equivalent to the given one, provided that $\operatorname{dim} M \leqq N$. Two sphere bundles over $M$ defined by the mappings $f_{i}(M) \subset H(n, N), i=1,2$, are equivalent, when and only when the mappings $f_{1}$ and $f_{2}$ are homotopic.

From the mapping $f(M) \subset H(n, N)$ the cocycles of dimension not greater than $\operatorname{dim} M$ of $H(n, N)$ are mapped by the inverse mapping into cocycles of $M$. The latter part of Theorem 8.1 asserts that the image cocycles of $M$ are cohomologous, if the sphere bundles are equivalent. The image of a cohomology class of $H(n, N)$ under the inverse mapping is therefore independent of the choice of the mapping $f$, provided that the sphere bundle induced remains equivalent. Such a cohomology class in $M$ is called a generalized characteristic class. 
If $M$ is a differentiable manifold and $\mathfrak{F}$ the tangent sphere bundle over $M$, we can find the mapping $f$ in question by imbedding $M$ in a Euclidean space $E^{n+N}$ and defining $f(P)$ to be the $n$-plane of $H(n, N)$ parallel to the tangent $n$-plane of $M$ at $P$. $M$ has then a Riemannian metric induced by the Euclidean metric of $E^{n+N}$.

In $E^{n+N}$ consider the frames $P \mathrm{e}_{1} \cdots \mathrm{e}_{n+N}$. We use the ranges of indices

$$
1 \leqq i, k \leqq n, \quad n+1 \leqq r, s \leqq n+N, \quad 1 \leqq A, B, C \leqq n+N,
$$

and put

$$
\omega_{A B}=d e_{A} \cdot e_{B} .
$$

Then we have

$$
d \omega_{A B}=\sum_{C} \omega_{A C \omega_{C B}}
$$

To each $n$-plane of $H(n, N)$ we attach the frames $O e_{1} \cdots e_{n} e_{n+1} \cdots$ $\mathrm{e}_{n+N}$ such that $\mathrm{e}_{1}, \cdots, \mathrm{e}_{n}$ belong to the $n$-plane. Then the $n N$ linear differential forms $\omega_{\alpha r}$ constitute a set of linearly independent forms of $H(n, N)$. To represent an integral cocycle of $H(n, N)$ in the sense of $\$ 7$ we define

$$
\Omega_{i k}=-\sum_{r} \omega_{i r} \omega_{k r}
$$

It is then a consequence of the first main theorem in the theory of vector invariants for orthogonal groups [31] that every integral cocycle of dimension not greater than $n$ of $H(n, N)$ can be represented by a differential form which is a linear combination, with constant coefficients, of the products of the forms $\Delta_{m}, \Delta_{0}$ defined by (64), where the $\Omega_{\imath k}$ are here given by (65).

The forms $\Omega_{i k}$ defined in (65) are forms of $H(n, N)$. But their images in $M$ induced by the mapping $f$ are the curvature forms of $M$ derived from the Riemannian metric of $M$ induced by $E^{n+N}$. In fact, let us attach to each point $P$ of $M$ all frames $P \mathrm{e}_{1} \cdots \mathrm{e}_{n+N}$ such that $\mathrm{e}_{1}, \cdots, \mathrm{e}_{n}$ are tangent vectors. On $M$ we have then

$$
\omega_{r}=0 \text {, }
$$

and

$$
\begin{aligned}
d \omega_{i} & =\sum_{k} \omega_{k} \omega_{k i}, \\
d \omega_{i k} & =\sum_{j} \omega_{i j} \omega_{j k}-\sum_{r} \omega_{i r} \omega_{k r}=\sum_{j} \omega_{i j} \omega_{j k}+\Omega_{i k} .
\end{aligned}
$$


From (66) it is seen that the images of the $\Omega_{i k}$ in (65) are the curvature forms of the Riemannian metric induced on $M$.

In this way we can prove the following theorem of Pontrjagin:

THEOREM 8.2. Let $M$ be a compact orientable differentiable manifold imbedded in a Euclidean space and let $\Omega_{i k}$ be the curvature forms derived from the induced Riemannian metric. An integral characterisic cocycle of $M$ can be represented by a differential form which is a linear combination, with constant coefficients, of the products of the forms $\Delta_{m}$, $0 \leqq m \leqq n / 4$.

It is highly probable that the relations asserted in Theorem 8.2 remain true, if the $\Omega_{i k}$ are the curvature forms of a Riemannian metric given intrinsically on $M$. But so far this has only been established for the case of the form $\Delta_{0}$ and for a few other cases. The result will follow if it can be proved that a Riemannian manifold can be isometrically imbedded in a Euclidean space. Unfortunately this imbedding question is not settled, even for $n=2$.

9. Hermitian geometry. Our results are more satisfactory in the case of Hermitian geometry. By an Hermitian manifold $M$ we shall mean a complex analytic manifold in which there is given an Hermitian metric:

$$
d s^{2}=\sum_{i, k=1}^{n} g_{i k}(z, \bar{z})\left(d z_{i} d \bar{z}_{k}\right), \quad \quad \bar{g}_{i k}=g_{k i},
$$

where $z_{i}$ are the local complex coordinates and where the bar denotes the operation of taking the conjugate complex.

As in the case of Riemannian manifolds, the principal fibre bundle for an Hermitian manifold is one whose fibres consist of a set of complex vectors $e_{1}, \cdots, e_{n}$, such that

$$
\mathrm{e}_{i} \cdot \overline{\mathrm{e}}_{j}=\delta_{i j},
$$

where the scalar product is understood in the sense of Hermitian geometry. Like the Riemannian case, the fundamental formulas of Hermitian geometry can be established to be

$$
d s^{2}=\sum\left(\omega_{i} \bar{\omega}_{i}\right), \quad d \omega_{i}=\sum \omega_{j} \omega_{j i}, \quad d \omega_{i k}=\sum \omega_{i j} \omega_{j k}+\Omega_{i k},
$$

where

$$
\omega_{i j}+\bar{\omega}_{j i}=0, \quad \Omega_{i j}+\bar{\Omega}_{j i}=0, \quad \Omega_{i j}=\sum_{k, l} R_{i j, k l} \omega_{k} \bar{\omega}_{l} .
$$

From $\Omega_{i j}$ we construct the differential forms 


$$
\Delta_{m}=\sum_{i_{1}, \cdots, i_{m}} \Omega_{i_{1} i_{2}} \Omega_{i_{2} i_{3}} \cdots \Omega_{i_{m} i_{1}}, \quad 1 \leqq m \leqq n,
$$

and also the differential forms

$$
\Psi_{m}=\sum \delta\left(i_{1}, \cdots, i_{m} ; j_{1}, \cdots, j_{m}\right) \Omega_{i_{1} j_{1}} \cdots \Omega_{i_{m} j_{m}}, \quad 1 \leqq m \leqq n,
$$

where $\delta\left(i_{1}, \cdots, i_{m} ; j_{1}, \cdots, j_{m}\right)$ is the Kronecker symbol which is equal to +1 or -1 according as $j_{1}, \cdots, j_{m}$ constitutes, an even or odd permutation of $i_{1}, \cdots, i_{m}$, and is otherwise zero, and where the summation is extended to all $i_{1}, \cdots, i_{m}$ from 1 to $n$. It is easy to see that every $\Psi_{m}$ can be expressed as a polynomial of $\Delta_{1}, \cdots, \Delta_{m}$ with constant coefficients, and conversely.

Over the compact Hermitian manifold $M$ we can construct the fibre bundle $\mathfrak{F}^{(p)}$ such that the fibre over a point $P$ of $M$ consists of ordered sets of vectors $\mathfrak{e}_{1}, \cdots, \mathfrak{e}_{p}$, satisfying the relations

$$
\mathfrak{e}_{i}-\overline{\mathrm{e}}_{j}=\delta_{i j}, \quad 1 \leqq i, j \leqq p .
$$

Then we can prove the following theorem:

THEOREM 9.1. The characteristic cocycle of $\mathfrak{F}^{(p)}$ in $M$ in the sense of $\$ 4$ is of dimension $2 n-2 p+2$ and can be defined, up to a constant factor, by the form $\Psi_{n-p+1}$.

These characteristic cocycles, altogether $n$ of them, corresponding to the values $p=1, \cdots, n$, we shall call the basic characteristic cocycles. On the other hand, the imbedding process of $\$ 8$ can be carried over to the present case, resulting in a correspondence between the equivalent fibre bundles over $M$ (in the sense of Steenrod) and the homotopy classes of mappings of $M$ into $H(n, N, C)$, where $H(n, N, C)$ is the Grassmann manifold of all $n$-dimensional complex linear spaces through the origin in a complex Euclidean space of $n+N$ (complex) dimensions. This allows us to define a generalized characteristic cohomology class of $M$ as the inverse image of a cohomology class of dimension not greater than $2 n$ of $H(n, N, C)$. But in the complex case the first main theorem on vector invariants for the unitary group is of simpler form than the corresponding theorem for the orthogonal group, and we can prove the following theorem:

THEOREM 9.2. Every cohomology class of $H(n, N, C)$ of dimension not greater than $2 n$ can be obtained by operations of the cohomology ring from $n$ basic classes, namely, the classes whose inverse images are the basic characteristic classes of $M$.

It is in this sense that we can say that for complex analytic Hermitian manifolds the characteristic classes arising from the con- 
sideration of the principal fibre bundle are completely determined by the Hermitian metric. We also remark that complex analytic Hermitian manifolds play an important rôle in algebraic geometry and in the theory of analytic functions of several complex variables.

The proofs of the results announced in this section will be published elsewhere.

10. The kinematic principal formula in integral geometry. In the above sections we have discussed the interrelations between local differential-geometric properties and topology. There are, however, other geometric properties in the large which are equally of interest and which are closely related to the ideas set forth above. Moreover, they also illustrate the important rôle played by the fibre bundle in the theory of a geometric being.

Let $M$ be a Riemannian manifold of $n$ dimensions, and let $₹$ be its principal fibre bundle. We have defined in the space $\mathfrak{F}$ of dimension $n(n+1) / 2$ the forms $\omega_{i}, \omega_{i k}$. The differential form of degree $n(n+1) / 2$ :

$$
\mathfrak{P}=\prod \omega_{i} \cdot \prod_{i<k} \omega_{i k}
$$

can serve as a kind of volume element in $\mathfrak{F}$. If $M$ is the Euclidean space of $n$ dimensions, $\mathfrak{B}$ is called the kinematic density, which was first introduced by Poincare for the case $n=2$.

To illustrate how the kinematic density can be utilized in geometrical problems suppose $M$ to be the Euclidean space of $n$ dimensions. Then $\mathfrak{F}$ is the space of frames in $M$ and is topologically a product. In $M$ we consider two closed hypersurfaces $S_{1}, S_{2}$ of class not less than 2 . For each $S_{i}$ let $r_{1}^{(i)}, \cdots, r_{n-1}^{(i)}$ be the principal curvatures and let

$$
\frac{1}{C_{n-1, k}} \int_{S_{i}} \sum r_{1}^{(i)} \cdots r_{k}^{(i)} d O_{i}=H_{k}^{(i)}, i=1,2 ; k=0, \cdots, n-1 \text {, }
$$

where the integrand is the $k$ th elementary symmetric function of the principal curvatures and $d O_{i}$ is the surface element of $S_{i}$. Denote by $V^{(i)}$ the volume bounded by $S_{i}$ in $M$. Let $S_{1}$ be fixed in the space and let $S_{2}$ take all possible positions. For each position of $S_{2}$ denote by $\chi\left(S_{1} S_{2}\right)$ the Euler-Poincaré characteristic of the intersection of $S_{1}, S_{2}$. Then we have the following so-called kinematic principal formula:

$$
\begin{aligned}
\int_{S_{2}} \chi\left(S_{1} \cdot S_{2}\right) \Re=J_{n}\left\{H_{n-1}^{(1)} V^{(2)}\right. & +V^{(1)} H_{n-1}^{(2)} \\
+ & \left.\frac{1}{n} \sum_{k=0}^{n-2} C_{n, k+1} H_{k}^{(1)} H_{n-2-k}^{(2)}\right\},
\end{aligned}
$$


where $J_{n}$ is the kinematic measure about a point in $M$ and is given by

$$
J_{n}=O_{n-1} O_{n-2} \cdots O_{1},
$$

$O_{p-1}$ being the area of the unit sphere in a Euclidean space of $p$ dimensions.

The formula (76) was proved by Blaschke for $n=2,3$ [2], and was established for general $n$ by Yien and the present writer [13]. It has numerous interesting consequences. In particular, when $n=2$ and when both $S_{1}$ and $S_{2}$ are convex curves, it becomes

$$
\int_{S_{1} \cdot S_{2} \times 0} \mathfrak{P}=2 \pi\left(A_{1}+A_{2}\right)+L_{1} L_{2},
$$

where $A_{i}$ is the area bounded by $S_{i}$ and $L_{i}$ is the length of $S_{i}, i=1,2$. Formula (77) was first established by Santalò and can be used to derive a sharpening of the isoperimetric inequality (2) [23]. It is thus through all the round-about discussions on differential geometry and topology that we arrived at the relation between the theory of fibre bundles and the isoperimetric inequality given at the beginning of this article.

\section{BIBLIOGRAPHY}

1. Allendoerfer, C. B., and Weil, A., The Gauss-Bonnet theorem for Riemannian polyhedra, Trans. Amer. Math. Soc. vol. 53 (1943) pp. 101-129.

2. Blaschke, W., Vorlesungen ibber Integralgeometrie, Part 1, 2d ed., Leipzig, 1936; Part 2, Leipzig, 1937.

3. Cartan, E., Les sousgroupes des groupes continus de transformations, Ann. Ecole Norm. (3) vol. 25 (1908) pp. 57-194.

4. - La theorie des groupes finis et continus et la géométrie différentielle traité par la methode du repère mobile, Paris, 1937.

5. - Leçons sur les invariants intégraux, Paris, 1922.

6. - Sur les variêtés a connexion affine et la theorie de la relativité généralisé, Ann. Ecole Norm. (3) vol. 40 (1923) pp. 325-412; vol. 41 (1924) pp. 1-25; vol. 42 (1925) pp. 17-88.

7. - Le principe de dualit et certaines intégrales multiples de l'espace tangentiel et de l'espace réglé, Bull. Soc. Math. France vol. 24 (1896) pp. 140-177.

8. - Sur les variêtés a connexion projective, Bull. Soc. Math. France vol. 52 (1924) pp. 205-241.

9. - Sur les invariants intégraux de certains espaces homogènes clos et les propriétés topologiques de ces espaces, Annales de la Société Polonaise de Mathématique vol. 8 (1929) pp. 181-225.

10. Chern, S., A simple intrinsic proof of the Gauss-Bonnet formula for closed Riemannian manifolds, Ann. of Math. vol. 45 (1944) pp. 747-752.

11. - Integral formulas for the characteristic classes of sphere bundles, Proc. Nat. Acad. Sci. U.S.A. vol. 30 (1944) pp. 269-273.

12. - A generalization of the projective geometry of linear spaces, Proc. Nat. Acad. Sci. U.S.A. vol. 29 (1943) pp. 38-43. 
13. Chern, S., and Yien, C. T., Sulla formula principale cinematica dello spazio ad $n$ dimensioni, Bollettino della Unione Matematica Italiana (2) vol. 2 (1940) pp. 432437.

14. Chern, S., On Riemannian manifolds of four dimensions, Bull. Amer. Math. Soc. vol. 51 (1945) pp. 964-971.

15. Ehresmann, C., Sur la topologie de certains espaces homogènes, Ann. of Math. vol. 35 (1934) pp. 396-443.

16. - Various notes on fibre spaces in C. R. Acad. Sci. Paris vol. 213 (1941) pp. 762-764; vol. 214 (1942) pp. 144-147; vol. 216 (1943) pp. 628-630.

17. Ehresmann, C., and Feldbau, J., Sur les proprietes d'homotopie des espaces fibres, C. R. Acad. Sci. Paris vol. 212 (1941) pp. 945-948.

18. Feldbau, J. See Ehresmann, C.

19. Paris Seminar, Notes on Elie Cartan's scientific work, Paris, 1936.

20. Pontrjagin, L., Characteristic cycles on manifolds, C. R. (Doklady) Acad. Sci. USSR N.S. vol. 35 (1942) pp. 34-37.

21. - On some topologic invariants of Riemannian manifolds, C. R. (Doklady) Acad. Sci. USSR N.S. vol. 43 (1944) pp. 91-94.

22. de Rham, G., Sur l'analysis situs des varietes a $n$ dimensions, J. Math. Pures Appl. vol. 10 (1931) pp. 115-200.

23. Santalò, L. A., Sobre la medida cinematica en el plano, Abh. Math. Sem. Hamburgischen Univ. vol. 11 (1935) pp. 222-236.

24. Steenrod, N., Topological methods for the construction of tensor functions, Ann. of Math. vol. 43 (1942) pp. 116-131.

25. - The classification of sphere bundles, Ann. of Math. vol. 45 (1944) D 294-311.

26. Stiefel, E., Richtungsfelder und Fernparallelismus in n-dimensionalen Mannigfaltigkeiten, Comment. Math. Helv. vol. 8 (1936) pp. 305-343.

27. Thomas, T.Y., and Veblen, O., The geometry of paths, Trans. Amer. Math. Soc. vol. 25 (1923) pp. 551-608.

28. Veblen, O. See Thomas, T. Y.

29. Veblen, O., and Whitehead, J. H. C., Foundations of differential geometry, Cambridge, 1932.

30. Weil, A. See Allendoerfer, C. B.

31. Weyl, H., The classical groups, Princeton, 1939.

32. Whitehead, J. H. C. See Veblen, O.

33. Whitney, H., Topological properties of differentiable manifolds, Bull. Amer. Math. Soc. vol. 43 (1937) pp. 785-805.

34. - On the topology of differentiable manifolds, Lectures in topology, Michigan, 1941, pp. 101-141.

35. Yien, C. T. See Chern; S.

INSTITUTE FOR ADVANCED STUdY AND

TSING HUA UNIVERSITY 